Article

\title{
Supervised Learning for Predictive Pore Size Classification of Regenerated Cellulose Membranes Based on Atomic Force Microscopy Measurements
}

\author{
Alex Hadsell ${ }^{1}$, Huong Chau ${ }^{1,2}$, Richard Barber, Jr. ${ }^{2,3}$, Unyoung Kim ${ }^{1,2}$ and Maryam Mobed-Miremadi ${ }^{1, *(\mathbb{D}}$ \\ 1 Department of Bioengineering, Santa Clara University, Santa Clara, CA 95053, USA; ahadsell@scu.edu (A.H.); \\ hchau@alumni.scu.edu (H.C.); ukim@scu.edu (U.K.) \\ 2 Center for Nanostructures, Santa Clara University, Santa Clara, CA 95053, USA; rbarber@scu.edu \\ 3 Department of Physics, Santa Clara University, Santa Clara, CA 95053, USA \\ * Correspondence: mmobedmiremadi@scu.edu; Tel.: +1-408-554-2731
}

check for

updates

Citation: Hadsell, A.; Chau, H.; Barber, R., Jr.; Kim, U.; Mobed-Miremadi, M. Supervised Learning for Predictive Pore Size Classification of Regenerated Cellulose Membranes Based on Atomic Force Microscopy Measurements. Materials 2021, 14, 6724. https:// doi.org/10.3390/ma14216724

Academic Editors: Claudio Canale and Christian Müller

Received: 11 September 2021 Accepted: 4 November 2021 Published: 8 November 2021

Publisher's Note: MDPI stays neutral with regard to jurisdictional claims in published maps and institutional affiliations.

Copyright: (c) 2021 by the authors. Licensee MDPI, Basel, Switzerland. This article is an open access article distributed under the terms and conditions of the Creative Commons Attribution (CC BY) license (https:/ / creativecommons.org/licenses/by/ $4.0 /)$.

\begin{abstract}
Nanoporous dialysis membranes made of regenerated cellulose are used as molecular weight cutoff standards in bioseparations. In this study, mesoporous standards with Stokes' radii $(50 \mathrm{kDa} / 2.7 \mathrm{~nm}, 100 \mathrm{kDa} / 3.4 \mathrm{~nm}$ and $1000 \mathrm{kDa} / 7.3 \mathrm{~nm})$ and overlapping skewed distributions were characterized using AFM, with the specific aim of generating pore size classifiers for biomimetic membranes using supervised learning. Gamma transformation was used prior to conducting discriminant analysis in terms of the area under the receiver operating curve (AUC) and classification accuracy (Acc). Monte Carlo simulations were run to generate datasets $(n=10)$ on which logistic regression was conducted using a constant ratio of 80:20 (measurement:algorithm training), followed by algorithm validation by WEKA. The proposed algorithm can classify the $1000 \mathrm{kDa}$ vs. $100 \mathrm{kDa}$ (AUC > 0.8) correctly, but discrimination is weak for the $100 \mathrm{kDa}$ vs. $50 \mathrm{kDa}$ (AUC < 0.7), the latter being attributed to the instrument accuracy errors below $5 \mathrm{~nm}$. As indicated by the results of the cross-validation study, a test size equivalent to $70 \%\left(\mathrm{AUC}_{\text {tapping }}=0.8341 \pm 0.0519, \mathrm{Acc}_{\text {tapping }}=76.8 \% \pm 5.9 \%\right.$ ) and $80 \%\left(\mathrm{AUC}_{\text {fluid }}=0.7614 \pm 0.0314, \mathrm{Acc}_{\text {tfluid }}=76.2 \% \pm 1.0 \%\right)$ of the training sets for the tapping and fluid modes are needed for correct classification, resulting in predicted reduction of scan times.
\end{abstract}

Keywords: supervised learning; atomic force microscopy; regenerated cellulose

\section{Introduction}

Cellulose is the most abundant biopolymer, with the common formula $\left(\mathrm{C}_{6} \mathrm{H}_{10} \mathrm{O}_{5}\right)_{n}$, consisting of a linear chain of $\beta-1,4$-glycosidic bonds with varying degrees of polymerization. Cellulose-based biomaterials are used in sustainability research [1,2], nanofiber research [3,4] and tissue engineering [5-7], amongst other applications. Regenerated cellulose (RC) membranes are hydrophilic, solvent resistant and non-woven, with multi-scale pore sizes ranging from $\mathrm{nm}$ to $\mu \mathrm{m}$. Across multiple reported porosities associated with bioseparation applications, the nanoscale pore size is used in hemodialysis [8] and bioprocessing $[9,10]$. Using cryopycnometry, it was shown that cyclic drying and rewetting generated a narrow skewed unimodal pore size pattern, with average pore sizes ranging from 5 to $10 \mathrm{~nm}$ over a range of modulated crystallinities [11]. Using scanning electron microscopy (SEM), it has been shown that RC membranes exhibited a homogenous macroand micro-porous structure on the surface, and the inner membrane ranged from 312 to $523 \mathrm{~nm}$ for the surface and from 187 to $320 \mathrm{~nm}$ for the cross-section [12]. RC membranes characterized by a molecular weight cutoff (MWCO) of $20 \mathrm{kDa}$ were used as standards for measuring the pore sizes of cross-linked alginate membranes using atomic force microscopy (AFM) [13].

Machine learning models that automatically generate descriptors that capture a complex representation of a material's morphology and structure have been developed for 
porous material characterization [14-17]. Specifically, a random forest-based machine learning model was generated based on AFM-based roughness characterization of crosslinked nanocellulose films' surfaces [18]. A major application of machine learning with AFM is for the correction and cleaning of AFM data [19-21]. Force-distance (F-D) data can be obtained from AFM scans, but indentation, baseline tilt, offset at contact point and other deviations result in rough curves. Using support vector machines and decision trees, these issues can be corrected for, resulting in smoother curves that are easier to analyze [1]. Similarly, neural networks have demonstrated the ability to increase the resolution and clarity of AFM images, allowing for easier analysis and presentation [20,21]. Algorithms have also shown an ability to remove simulated artifacts from AFM images [22]. Some convolutional neural networks are even capable of generating AFM images from scratch [21]. Using F-D curves generated by an AFM and a neural network, researchers were able to distinguish between glioblastoma and multiple myeloma cells from healthy cells [23]. Similarly, using AFM data and random forests, researchers could distinguish between cancerous and noncancerous cells found in urine with $90 \%$ accuracy [24]. The other major application of machine learning and AFM is for classification of the material being scanned by the AFM [22-27].

As captured in Table 1, over the breadth of biological samples, a critical aspect of the data used in these analyses is the resolution of the AFM. In cases where the image processing software, instrument calibration and sample fabrication are ruled out as sources of error, analysis of artifacts may be limited to spatial heterogeneity and probe aspect ratio.

Table 1. Materials characterized using AFM and machine learning.

\begin{tabular}{ccc}
\hline Material & $\begin{array}{c}\text { Machine Learning } \\
\text { Method(s) }\end{array}$ & Study \\
\hline Zebrafish spinal cord & $\begin{array}{c}\text { Support vector machines } \\
\text { Decision trees }\end{array}$ & Müller, et al. [19] \\
\hline $\begin{array}{c}\text { Glioblastoma and multiple myeloma cells } \\
\text { Cells found in urine }\end{array}$ & Random forests, extremely randomized forests, gradient \\
boosting trees & Sokolov, et al. [24] \\
\hline $\begin{array}{c}\text { Ferritic electric materials and } \\
\text { electrochemical systems }\end{array}$ & Support vector machines & Huang, et al. [25] \\
\hline $\begin{array}{c}\text { Ionic liquid layers on top of graphite and } \\
\text { melem on boron nitride }\end{array}$ & $\begin{array}{c}\text { Fast-Fourier Transform (FFT), primary component analysis } \\
\text { (PCA), cross-correlation (CC) and stationary wavelet } \\
\text { decomposition }\end{array}$ & Borodinov, et al. [26] \\
\hline DNA fragments & Linear regression & Sundstrom, et al. [27] \\
\hline $\begin{array}{c}\text { Human leukemia cells } \\
\text { AFM images with simulated artifacts }\end{array}$ & $\begin{array}{c}\text { Support vector machines and linear discriminant analysis } \\
\text { Low-resolution AFM images }\end{array}$ & Mencattini, et al. [22] \\
\hline Simulated AFM images & Convolutional Neural Network (CNN) & Liu, et al. [20] \\
\hline
\end{tabular}

The first step in biomimetic membrane design is theoretical flux calculations subject to unquantified effects, namely biofouling, concentration polarization hydrophobic interactions and electrostatic interactions. The second step is the choice of bio-fabrication method and associated materials' interaction, often resulting in non-ideal networks, uneven shrinkage with sub-optimal pore sizes and surface pores instead of through pores, amongst other post-processing artifacts. The last step is the choice of scanning probe microscopy method resolution with regards to the sample's intensive properties. Use of commercial RC membranes circumvents the last two challenges. Thus, the research objectives of this study are three-fold: (1) To establish a computational framework for generating pore size classifications for RC-based dialysis membranes as a model system based on discriminant analysis, as evaluated by the receiver operating characteristics curve (ROC), namely the 
area under the curve (AUC) and classification accuracy (Acc). (2) To assess the effect of sample size reduction for a reduction of scan time on classification quality based on a constant 80:20 training to test ratio, for the bootstrapped datasets evaluated using the holdout sub-sampling technique [28]. (3) To detect instrument accuracy errors using the above-mentioned supervised learning methodology. On the basis of this proposed algorithm, pore size calibration using certified MWCO within the range of the theoretical Stokes' radii can be achieved. In further steps, images of synthesized membranes can be superimposed to the calibration images for discriminant analysis, with a predicted pore size range characterized by a given classification accuracy as output.

\section{Results}

\subsection{Baseline Diffusion Measurements}

Shown in Table 2 are the equilibrium absorbance differentials (A- $\left.\mathrm{A}_{0}\right)$ across MWCOs. All values exceed zero, thus confirming the hypothesis of outward diffusion of the marker from the membrane and the presence of through pores.

Table 2. Diffusion results evaluated by spectrophotometry.

\begin{tabular}{cc}
\hline Marker/Membrane MWCO & $\left(\mathbf{A}-\mathbf{A}_{\mathbf{0}}\right)$ \\
\hline $4 \mathrm{kDa} / 50 \mathrm{kDa}$ & 0.37 \\
\hline $4 \mathrm{kDa} / 100 \mathrm{kDa}$ & 0.61 \\
\hline $4 \mathrm{kDa} / 1000 \mathrm{kDa}$ & 1.13 \\
\hline
\end{tabular}

\subsection{Atomic Force Microscopy}

The accuracy error for the calibration standard in tapping mode was $1.4 \mathrm{~nm}$ (refer to Supplementary Figure S1). AFM imaging was performed on single ply dialysis tubing either hydrated in saline prior to or submerged throughout imaging for the tapping and fluid modes, respectively.

Shown in Figure 1a-d are representative 2D views with corresponding insets for multiple scan areas, displaying skewness across all samples examined.

For the $1000 \mathrm{kDa}$, switching from tapping (Figure 1a) to fluid mode (Figure 1b) enabled capture of additional data points due to membrane hydration and improved resolution in order to focus into the deepest areas representing the smallest opening of the pores. For the $100 \mathrm{kDa}$ fluid mode, hydration capture was equally beneficial, however for the $50 \mathrm{kDa}$ sample, $4 \mu \mathrm{m}^{2}$ scan areas produced blurry images (tapping mode images are not shown). These results provide a benchmark for the instrument capabilities in an attempt to correlate these findings with the classification analyses that follow.

Random sampling of pore radii across the single ply dialysis membrane resulted in 12 pores/section and 76 pores/section for the tapping and fluid modes. Combined results of the examination of instrument drift, environmental changes and statistical analysis of pore size measurements are presented in Table 3. As reflected in the absolute values of the adjusted coefficient of determination $\left(R_{a d j}^{2}\right)$ deviating from unity for the filtered and unfiltered data, the effect of block variables, namely instrument drift due the spatial distribution, is negligible. The hypothetical changes in environmental conditions comprised of pore shrinkage as a result of evaporation for the tapping mode and continuous swelling for the fluid mode can also be ruled out based on the non-statistically significant $p$-values exceeding 0.05 for the Kruskal-Wallis test. The raw data for this analysis is presented in Supplementary Figure S2A.

For both imaging modes, wide measurement ranges could account for skewed or multimodal pore size distributions, as demonstrated in the frequency charts presented in Supplementary Figure S2B. Relative errors of accuracy increase with decreasing pore sizes, indicative of a lower limit of detection resolution. Notably, for the fluid mode, the average size of the $50 \mathrm{kDa}$ membrane $(11.1 \mathrm{~nm})$ is larger than the $100 \mathrm{kDa}$ membrane $(8.1 \mathrm{~nm})$. For 
the $1000 \mathrm{kDa}$ sample characterized by the lowest relative accuracy measurement errors, the average size captured is $62 \%$ higher for the fluid than the one for the tapping mode. Although numerical results could show that the triangular cantilever geometry used in this study is preferable for AFM measurements in fluid due to lower drag forces [29], resonance challenges in fluid mode enhanced by the possible effect of sample tip contamination remain a source of bias between the two imaging modes.
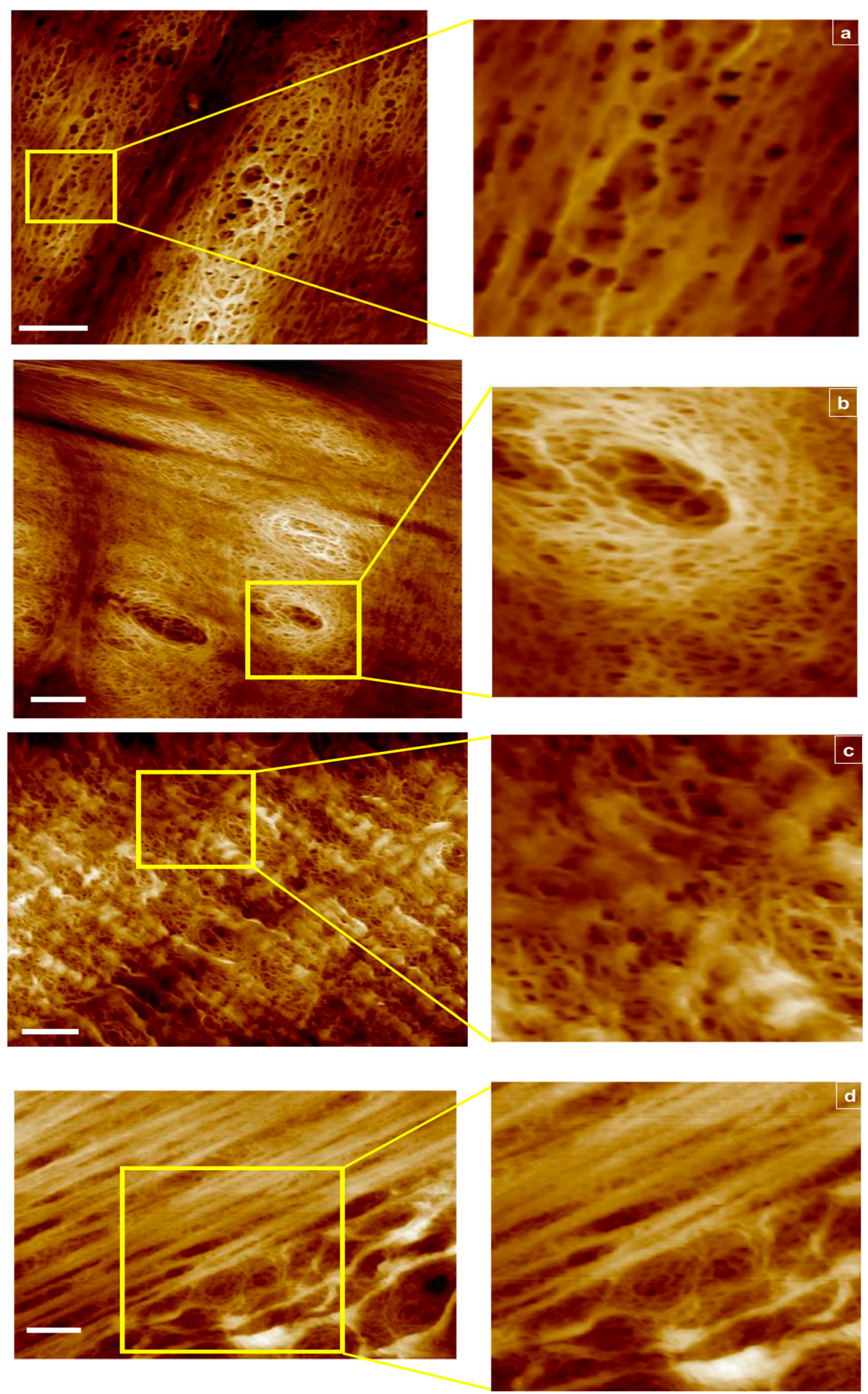

Figure 1. AFM images of RC membranes from top to bottom: (a) $1000 \mathrm{kDa}$ in tapping mode scan area $6 \mu \mathrm{m}^{2}, 2 \mu \mathrm{m}^{2}$ section magnified, (b) $1000 \mathrm{kDa}$ in fluid mode, scan area $8 \mu \mathrm{m}^{2}, 2 \mu \mathrm{m}^{2}$ section magnified, (c) $100 \mathrm{kDa}$ in fluid mode scan area $8 \mu \mathrm{m}^{2}, 2 \mu \mathrm{m}^{2}$ section magnified, and (d) $50 \mathrm{kDa}$ in fluid mode scan area $8 \mu \mathrm{m}^{2}, 4 \mu \mathrm{m}^{2}$ section magnified. Scale bar represents $1 \mu \mathrm{m}$. 
Table 3. Pore size measurements and associated errors of measurement accuracy.

\begin{tabular}{cccccc}
\hline Statistic & Mode & n & 1000 kDa & 100 kDa & 50 kDa \\
\hline Theoretical Stokes' radius (nm) & N/A & N/A & 7.3 & 3.4 & 2.7 \\
\hline Average (nm) & Tapping & 60 & 9.45 & 7.34 & 5.65 \\
\hline Measured range (nm) & Tapping & 60 & 23 & 41 & 19 \\
\hline \% relative error of accuracy & Tapping & 60 & 29.4 & 116 & 109 \\
\hline absolute error of accuracy (nm) & Tapping & 60 & 2 & 4 & 3 \\
\hline $\mathrm{R}_{\text {adj }}^{2}$ Fourier fit raw data & Tapping & 60 & 0.430 & 0.091 & 0.200 \\
\hline $\mathrm{R}_{\text {adj }}^{2}$ Fourier fit filtered data & Tapping & N/A & 0.290 & 0.212 & 0.380 \\
\hline Kruskal-Wallis test p-value & Tapping & 60 & 0.5384 & 0.9569 & 0.924 \\
\hline Average (nm) & Fluid & 304 & 15.3 & 8.34 & 11.1 \\
\hline Measured range (nm) & Fluid & 304 & 138 & 44 & 72 \\
\hline$\%$ relative error of accuracy & Fluid & 304 & 110 & 145 & 312 \\
\hline absolute error of accuracy (nm) & Fluid & 304 & 8 & 5 & 8 \\
\hline $\mathrm{R}_{\text {adj }}^{2}$ Fourier fit raw data & Fluid & 304 & 0.057 & 0.036 & 0.02 \\
\hline $\mathrm{R}_{\text {adj }}^{2}$ Fourier fit filtered data & Fluid & N/A & 0.398 & 0.371 & 0.450 \\
\hline Kruskal-Wallis test p-value & Fluid & 304 & 0.1534 & 0.681 & 0.681 \\
\hline
\end{tabular}

\subsection{Classifier Model Development}

2.3.1. Normality Assessment and Hypothesis Testing for Inter-MWCO Discrimination

As presented in Table 4, all datasets failed the normality test according to the Jarque and Bera metric $(J B)$ as the parameter value exceeds the critical value at the significance level of 0.05 . Hence, no statistical significance test was conducted using parametric methods.

Table 4. Summary of normality assessment conducted on raw datasets.

\begin{tabular}{|c|c|c|c|c|c|c|c|}
\hline RC Type & $\begin{array}{l}\text { Gaussian } \\
\text { Moments }\end{array}$ & Tapping $(n=60)$ & $J B *$ & $\begin{array}{c}\text { Normality } \\
\text { Assessment }\end{array}$ & Fluid $(n=304)$ & $J B * *$ & $\begin{array}{c}\text { Normality } \\
\text { Assessment }\end{array}$ \\
\hline \multirow[t]{2}{*}{$50 \mathrm{kDa}$} & Skewness & 2.069 & \multirow[t]{2}{*}{113} & \multirow[t]{2}{*}{ Fail } & 2.307 & \multirow[t]{2}{*}{733} & \multirow[t]{2}{*}{ Fail } \\
\hline & Kurtosis & 8.201 & & & 9.048 & & \\
\hline \multirow[t]{2}{*}{$100 \mathrm{kDa}$} & Skewness & 2.599 & \multirow[t]{2}{*}{371} & \multirow[t]{2}{*}{ Fail } & 2.833 & \multirow[t]{2}{*}{1545} & \multirow[t]{2}{*}{ Fail } \\
\hline & Kurtosis & 9.338 & & & 12.482 & & \\
\hline \multirow[t]{2}{*}{$1000 \mathrm{kDa}$} & Skewness & 2.314 & \multirow[t]{2}{*}{117} & \multirow[t]{2}{*}{ Fail } & 4.776 & \multirow[t]{2}{*}{17,919} & \multirow[t]{2}{*}{ Fail } \\
\hline & Kurtosis & 8.017 & & & 39.379 & & \\
\hline
\end{tabular}

$$
{ }^{*} J B_{\text {critical }}=5.1 ;{ }^{* *} J B_{\text {critical }}=5.7 \text {. }
$$

Results of the subsequent Kruskal-Wallis test summarized in Table 5 indicate that the AFM cannot resolve the difference between the $50 \mathrm{kDa}$ vs. $100 \mathrm{kDa}$ membranes for either imaging mode, as reflected by the $p$-values $(p>0.05)$. However, there is clear discrimination between $50 \mathrm{kDa}$ vs. $1000 \mathrm{kDa}(2.7 \mathrm{vs} .7 .3 \mathrm{~nm})$ and $100 \mathrm{kDa}$ vs. $1000 \mathrm{kDa}$ (3.4 nm vs. $7.3 \mathrm{~nm}$ ), as well as the corresponding theoretical Stokes' radii $(p<0.05)$.

In the following sections, logistic regression will be conducted post non-parametric analysis for predictive modeling of pore size and the associated goal of scan reduction time. 
Table 5. Hypothesis testing results for the Kruskal-Wallis test at the 95\% confidence interval.

\begin{tabular}{ccc}
\hline Mode & Comparison & $p$-Value \\
\hline Tapping & 100 vs. $1000 \mathrm{kDa}$ & 0.000 \\
Tapping & 50 vs. $1000 \mathrm{kDa}$ & 0.000 \\
Tapping & 50 vs. $100 \mathrm{kDa}$ & 0.275 \\
Fluid & 100 vs. $1000 \mathrm{kDa}$ & 0.000 \\
Fluid & 50 vs. $1000 \mathrm{kDa}$ & 0.000 \\
Fluid & 50 vs. $100 \mathrm{kDa}$ & 0.083 \\
\hline
\end{tabular}

\subsubsection{Algorithm Validation}

Shown in Table 6 is a comparison of AUCs by membrane/imaging mode pair based on logistic regression parameters, as well as corresponding WEKA outputs for the Gammatransformed datasets (refer to Supplementary Figure S2C). There is agreement between the results of the proposed methodology and the open-source software for all six combinations examined. The maximum calculated error of accuracy is $8 \%$ for $50 \mathrm{kDa}$ vs. $100 \mathrm{kDa}$ in tapping mode below the $10 \%$ threshold of rejection. Due the convergence of the results, the proposed algorithm has been validated against WEKA. For the $100 \mathrm{kDa} v \mathrm{vs} .1000 \mathrm{kDa}$ combination, an acceptable AUC was obtained for both imaging modes. For the $50 \mathrm{kDa}$ vs. $1000 \mathrm{kDa}$ combination, discrimination was stronger for the tapping vs. fluid mode. As for the $50 \mathrm{kDa}$ vs. $100 \mathrm{kDa}$ discrimination, it is not possible using either imaging mode.

Table 6. Comparison of algorithm-generated AUCs and WEKA outputs by imaging mode for a split ratio of 80:20 (training: test).

\begin{tabular}{cccc}
\hline Mode & Comparison & Weka AUC (80\%) & Algorithm AUC (80\%) \\
\hline Tapping & 100 vs. $1000 \mathrm{kDa}$ & 0.931 & $0.852 \pm 0.0335$ \\
Tapping & 50 vs. $1000 \mathrm{kDa}$ & 0.785 & $0.722 \pm 0.0424$ \\
Tapping & 50 vs. $100 \mathrm{kDa}$ & 0.590 & $0.604 \pm 0.0437$ \\
Fluid & 100 vs. $1000 \mathrm{kDa}$ & 0.753 & $0.762 \pm 0.0104$ \\
Fluid & 50 vs. $1000 \mathrm{kDa}$ & 0.663 & $0.661 \pm 0.0184$ \\
Fluid & 50 vs. $100 \mathrm{kDa}$ & 0.576 & $0.579 \pm 0.0172$ \\
\hline
\end{tabular}

In conjunction with increasing measurement accuracy errors associated with smaller pore sizes previously elaborated upon based on the data presented in Table 3, crossvalidation will be confined to $100 \mathrm{kDa}$ vs. $1000 \mathrm{kDa}$ comparisons.

\subsubsection{Cross-Validation and Minimum Sample Size Determination}

The AUC for the datasets as well as the logistic distribution parameters obtained at the optimal Youden indices for the datasets at 100\% sampling and optimal sample testing percentage, denoted by the subscript "opt", are presented in Table 7.

Table 7. Comparison of classifier strength at 70\% for tapping mode and $80 \%$ for fluid mode.

\begin{tabular}{cccccccc}
\hline Method & Comparison & \multirow{2}{*}{ AUC at 100\% } & AUC $_{\text {opt }}$ & $\begin{array}{c}\text { Youden at } \\
\mathbf{1 0 0 \%}\end{array}$ & Youden $_{\text {opt }}$ & $\boldsymbol{\beta}_{\mathbf{0 o p t}}$ & $\boldsymbol{\beta}_{\mathbf{1 0 p t}}$ \\
\hline Tapping & 100 vs. $1000 \mathrm{kDa}$ & $0.8521 \pm 0.0335$ & $0.8341 \pm 0.0519$ & $0.442 \pm 0.0496$ & $0.463 \pm 0.0582$ & $-4.1233 \pm 0.9533$ & $0.5998 \pm 0.1316$ \\
Fluid & 100 vs. $1000 \mathrm{kDa}$ & $0.7622 \pm 0.0104$ & $0.7614 \pm 0.0314$ & $0.287 \pm 0.0209$ & $0.294 \pm 0.0438$ & $-1.7784 \pm 0.2600$ & $0.1561 \pm 0.0256$ \\
\hline
\end{tabular}

The effect of testing data size on AUC and accuracy is presented in Figure 2a,b. For both imaging modes, the standard deviation of AUC decreases with increased bootstrapped data size. Based on the optimization criteria applied to the average AUC, a test size equivalent to $70 \%$ and $80 \%$ of the training sets for the tapping and fluid modes are recommended, respectively. Retrofitting these percentages to our experimental results in terms of sample size, the test to training ratios are 34:8 and 243:61. 

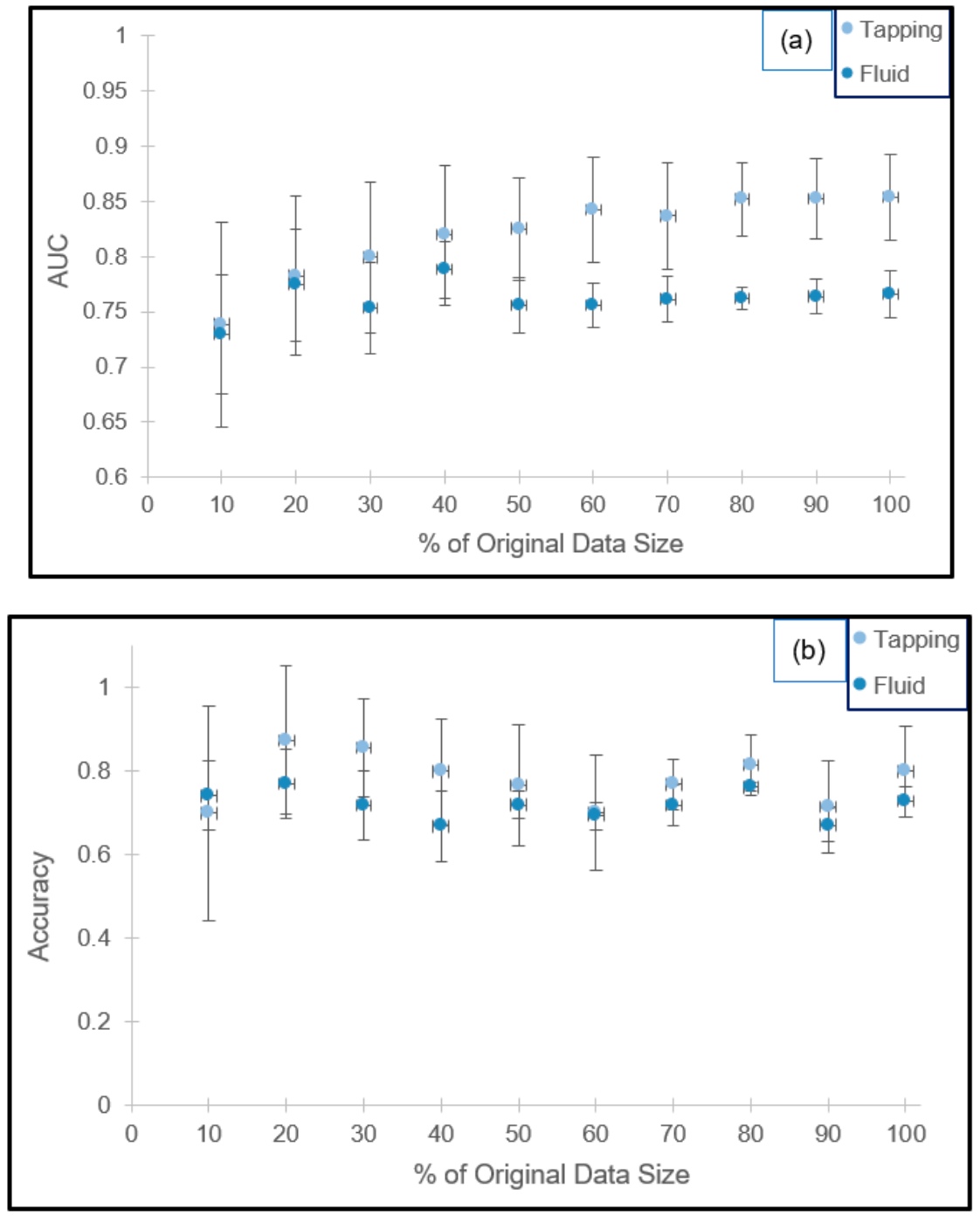

Figure 2. Cross-validation results for the $100 \mathrm{vs} .1000 \mathrm{kDa}$ combinations in tapping and fluid modes in terms of the AUC ((a) top) and classification accuracy ((b) bottom).

Furthermore, the standard deviations are an order of magnitude smaller than the average value $\left(\mathrm{AUC}_{\text {tapping }}=0.8341 \pm 0.0519\right.$ and $\left.\mathrm{AUC}_{\text {fluid }}=0.7614 \pm 0.0314\right)$ for the 10 randomly generated datasets, suggesting the absence of overfitting. Corresponding accuracies are $76.8 \% \pm 5.9 \%$ and $76.2 \% \pm 1.0 \%$, respectively.

Under these recommended settings, assuming a strong linear correlation between test size and scan time, the predicted AFM usage time is reduced by $30 \%$ and $20 \%$ for the tapping and fluid modes accordingly.

ROC curves resulting from 10 bootstrapped datasets generated using the optimal parameters in Table 7 as well the ones for the other comparisons for the same optimal settings (70\% for tapping and $80 \%$ for fluid) are presented in Figure 3a-e. Under this reduced sampling plan, visual inspection of the curves further confirms that discriminant analysis should be confined to the $1000 \mathrm{kDa}$ vs. $100 \mathrm{kDa}$. 


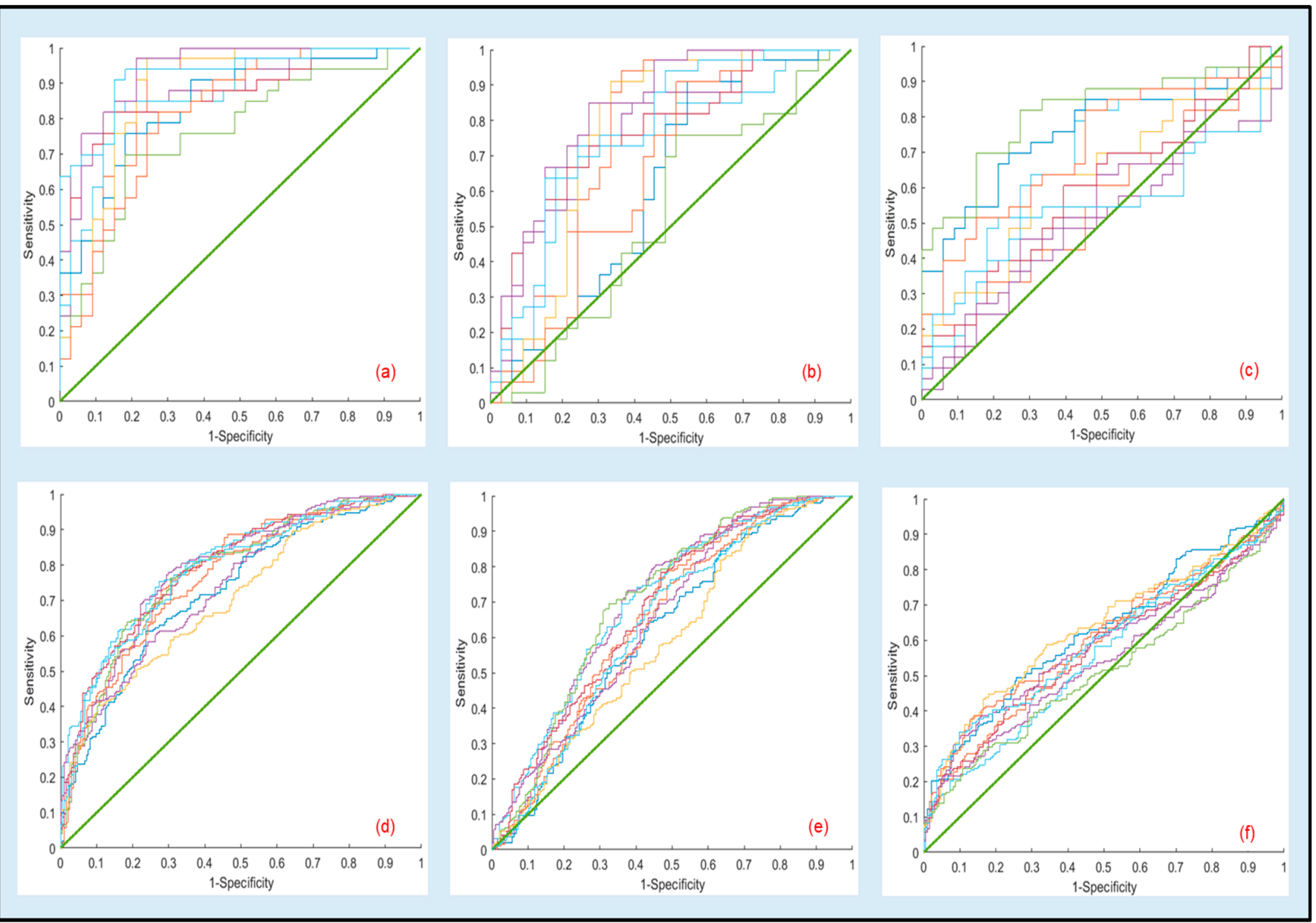

Figure 3. Representative ROC curves for the cross-validation results generated using logistic regression parameters from 10 bootstrapped datasets estimated at the optimal testing sizes of $70 \%$ for tapping and $80 \%$ for fluid. Top and bottom rows correspond to tapping and fluid modes, respectively: (a,d) $100 \mathrm{kDa} v \mathrm{~s} .1000 \mathrm{kDa}$ in tapping and fluid modes, (b,e) $50 \mathrm{kDa}$ vs. $1000 \mathrm{kDa}$ in tapping and fluid modes, (c,f) $50 \mathrm{kDa}$ vs. $100 \mathrm{kDa}$ in tapping and fluid modes. Regression parameters for $(\mathbf{a}, \mathbf{c})$ are presented in Table 7 .

ROC curves for the raw datasets are presented in Supplementary Figure S3.

\section{Discussion}

Given the multifactorial aspect of the study encompassing environmental factors, tip contamination, instrument capabilities and non-gaussian data, the following assumptions will be made based on the results before delving into the contribution of each factor.

The pore size of the RC membrane has been assumed to be the Stokes' radius $\left(r_{p}\right)$ calculated based on the MW of the manufacturer. Since the measurements have been conducted by the same individual, the magnitude of the reproducibility errors has been assumed to be negligible. As for instrument errors, periodic stage drifts have been ruled out due to poor adjusted coefficients of determination $\left(R_{a d j}^{2}<<1\right)$ of Fourier fits applied to the raw datasets (Supplementary Figure S2A). The null hypotheses of chronological and spatial environmental shifts, specifically membrane drying for tapping and continuous membrane swelling, have been accepted for all membrane/marker combinations based on the results of the Kruskal-Wallis test $(p>0.05)$.

Across expanding scanning probe characterization studies of cellulose nanomaterials using silicon nitride tips, encompassing contact resonance spectroscopy, chemical force spectroscopy (CFS) and atomic force microscope-based infrared spectroscopy (AFM-IR), tip contamination has been reported [30-33]. Root causes of contamination can be nar- 
rowed down to silicon residue from the PDMS storage container [34], surface adhesion of functionalized tips [30] and wear. Regarding the latter, associated root causes are chipping of metal-coated tips in Nano-IR [31,32] and tip contamination due to sample wear [33]. In the current study, precautions were taken to change the tip before each scan in order to minimize sample wear residue, however the tips were not cleaned prior to usage. Possible sources of contamination are silicon oil residue and dialysis membrane wear, resulting in decreased resolution for both imaging modes. For the tapping mode, the interaction volume between the probe and the surface is made larger by the contamination layer, while for the fluid mode, the balance of hydrodynamic forces may be disturbed by micelle formation or random residue deposits.

In the absence of statistically significant spatial and temporal shifts throughout the scan (Supplementary Figure S2A) along the use of non-functionalized and uncoated tips, it has been assumed that the unquantified effect of contamination is a source of systematic error.

Cellulose-based hydrogels are susceptible to swelling [35], with a reported swelling ratio (SR) ranging from $50 \%$ to $3000 \%$ for regenerated cellulose [36,37]. A range of $\mathrm{SR}=50-200 \%$ has been adapted to calculate the theoretical pore radius in order to interpret the departures from the manufactured values. The objective is to find an initial swelling ratio range based on a common direction of change for all three standards for measurement error minimization, assuming that the swelling rate is the same at all MWCOs. Shown in Figure 4 are the absolute errors of measurement accuracy adjusted to swelling ratios (SR) calculated based on the theoretical Stokes' radii. Between the ratios of $50-100 \%$, accuracy errors are decreasing in the same direction for all RC membranes. Specifically, for the 1000 and $100 \mathrm{kDa}$ membranes, the error is within $\pm 1.5 \mathrm{~nm}$ for SR $=75-100 \%$, in contrast to the $50 \mathrm{kDa}$ membrane with approximately double the error range $(6-7 \mathrm{~nm})$. For the latter, accuracy is inversely proportional to the swelling ratio, and an optimum of $3 \mathrm{~nm}$ is reached at $\mathrm{SR}=200 \%$. At this maximal projected swollen state, the $1000 \mathrm{kDa}$ measurement error is maximal, contradicting how the measured relative errors of accuracy increase with decreasing pore sizes for the fluid mode. Thus, initial hydrogel swelling state cannot account for the poor instrument resolution between the 100 and $50 \mathrm{kDa} R C$ membranes.

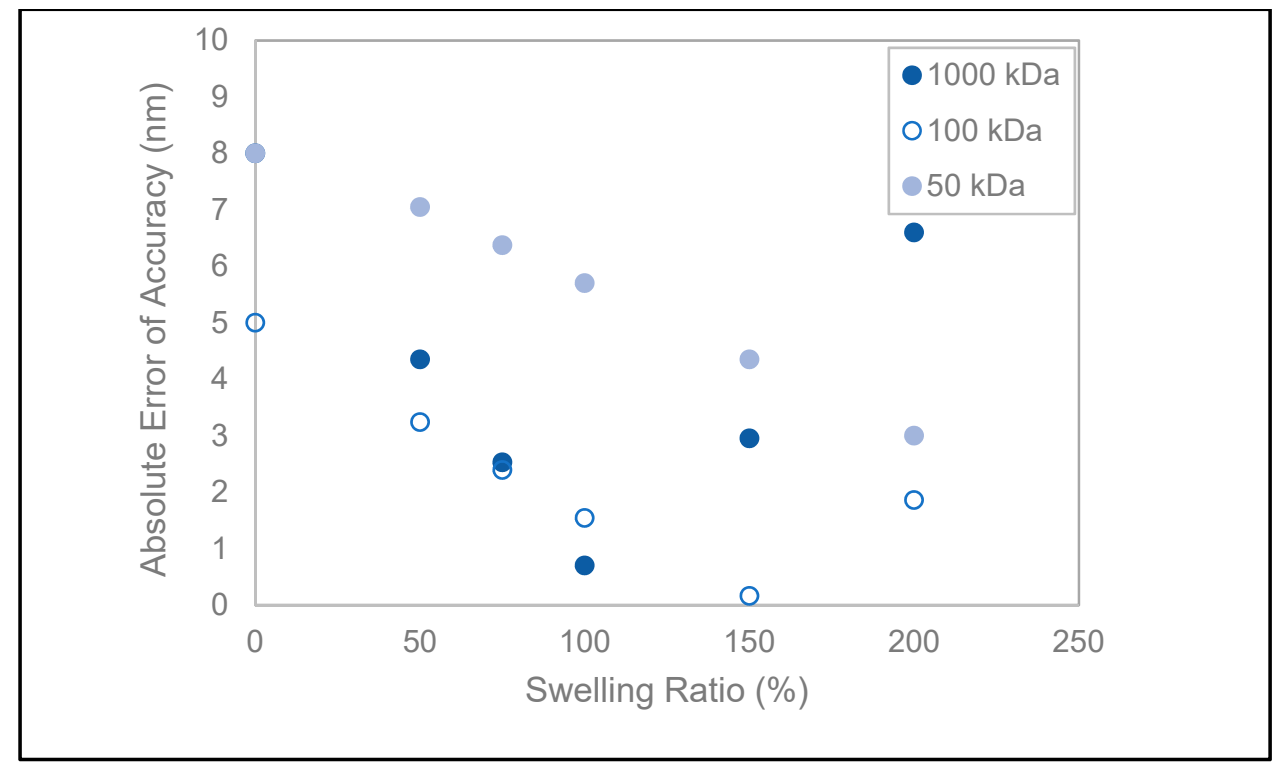

Figure 4. Absolute measurement accuracy error as a function of the hypothetical swelling ratio.

A critical aspect of the data used in these analyses is the spatial resolution of the AFM, in this study estimated to be $\pm 1.5 \mathrm{~nm}$ using the calibration step in tapping mode (refer to Supplementary Figure S1). Uncertainties in these measurements can have a significant impact on the discriminant analysis, especially when the smallest features are considered. 
In this study, the smallest error of accuracy of $2 \mathrm{~nm}$ is within the uncertainty range of the calibration step measured in tapping mode for the $1000 \mathrm{kDa}$ RC membrane. Results of another study conducted in tapping mode on the same membrane feature-extracted at $0.25 \mu \mathrm{m}^{2}$ instead of $1 \mu \mathrm{m}^{2}$-resulted in pore diameters ranging from 13 to $18 \mathrm{~nm}$ with no specified sample size, as compared to a range of 5-28 nm [38]. Theoretically, a decrease in scan area allows the ability to zoom into the deepest areas of the image, which represents the smallest opening of the pores assuming that the resolution of the scanner is adequate.

In order to determine the lateral resolution of an AFM, Moiseev et al. calculated the contribution to the van der Waals forces for various tip radii and tip-to-sample distances under ambient conditions. They found that for a tip radius of curvature of $5 \mathrm{~nm}$, lateral resolution could approach $2 \mathrm{~nm}$ if the tip was held very close $(0.5 \mathrm{~nm})$ to the surface [39]. A more detailed analysis by Gan concludes that even larger working distances can be impractical due to attractive forces pulling the tip to the surface. The main conclusion regarding lateral resolution is that the theoretical limit is strongly determined by the tip geometry [40]. Furthermore, given the instrument- and environment-dependent nature of AFM measurements, it is perhaps more fruitful to consider resolution results from experimental studies for tips with a radius of curvature of $10 \mathrm{~nm}$, typical of the NanoWorld PNP-TR tips utilized in this work [13,41], for which the best lateral resolution ranged between about 3 and $5 \mathrm{~nm}$. The most accurate result has been obtained for an RC membrane with a MWCO of $20 \mathrm{kDa}\left(r_{p}=2.02 \mathrm{~nm}\right)$. Using the tapping mode, an average pore diameter of $4.9 \mathrm{~nm}$ was measured corresponding to an accuracy error of $143 \%$, consistent with the obtained results for Stokes' radii below $5 \mathrm{~nm}$, as presented in Table 3 [13]. In another study, a direct resolution comparison was performed experimentally with double-wall carbon nanotubes (DWNTs) and a conventional $8 \mathrm{~nm}$ radius of curvature Si tips. The results indicated lateral resolutions of 5-6 nm for the DWNTs and 32-35 nm with the Si tips [42]. These results further support that the observations of the AFM instrument used in this work could not reliably resolve features below $5 \mathrm{~nm}$. AFM imaging in fluids using tapping mode is subject to resonance challenges. In water, the relatively low Q-factor (20-30) of the cantilever results in a degradation of spatial resolution [43]. A method for overcoming this challenge is frequency modulated AFM (FM-AFM) conducted in fluid and tapping modes [44]. Tip treatments can also improve the interaction of the tip with the sample, and atomic resolution is possible. Comparisons between untreated and treated tips show a dramatic resolution enhancement, with untreated tips producing lower quality images [42]

In this study, a sample size imbalance occurred between the two imaging modes, where 60 points vs. 304 points were sampled for the tapping and fluid modes, respectively. The lower sample size for the former was to prevent sample shrinkage at lower scan rates, while the higher sample sizes were designed to increase the power of the test, the latter being susceptible to resonance challenges [43]. Nevertheless, each dataset contained a minimum of 30 samples, maximizing the chances of the Central Limit Theorem to hold in the absence of skew.

Regardless of the fabrication and characterization method, the pore size distribution of RC membranes has been reported to be skewed, in agreement with the results of this study $[10,19,37]$. As shown in Table 8, there is agreement between AUCs generated from the raw datasets (refer to Supplementary Figure S3) and the those obtained from proposed logistic regression models at 100\% sampling, except for the combination F2. In future studies with enhanced instrumentation capability, outlier analysis will be conducted to further examine the extent of the inherent sample skewness incorporated into algorithm development. 
Table 8. Comparison of classifier agreement between raw datasets and the proposed algorithm.

\begin{tabular}{ccccc}
\hline Combination & Comparison & $\begin{array}{c}\text { Raw Data } \\
\text { AUC }\end{array}$ & $\begin{array}{c}\text { Algorithm } \\
\text { AUC (100\%) }\end{array}$ & Agreement \\
\hline T1 & 100 kDa vs. $1000 \mathrm{kDa}$ & 0.894 & $0.8521 \pm 0.0335$ & Yes \\
T2 & $50 \mathrm{kDa}$ vs. $1000 \mathrm{kDa}$ & 0.749 & $0.7216 \pm 0.0425$ & Yes \\
T3 & $50 \mathrm{kDa}$ vs. $100 \mathrm{kDa}$ & 0.620 & $0.6035 \pm 0.04366$ & Yes \\
F1 & $100 \mathrm{kDa}$ vs. $1000 \mathrm{kDa}$ & 0.794 & $0.7622 \pm 0.0104$ & Yes \\
F2 & $50 \mathrm{kDa}$ vs. $1000 \mathrm{kDa}$ & 0.708 & $0.6613 \pm 0.0184$ & No \\
F3 & 50 kDa vs. $100 \mathrm{kDa}$ & 0.541 & $0.5821 \pm 0.0385$ & Yes \\
\hline
\end{tabular}

\section{Materials and Methods}

\subsection{Materials}

Spectrum $^{\mathrm{TM}}$ Labs Regenerated Cellulose (RC) dialysis membranes with molecular weight cutoffs (MWCO)s of 50 kDa (08-700-128), 100 kDa (08-700-132) and $1000 \mathrm{kDa}(08-$ 801-255) were purchased from Fisher Scientific (Waltham, MA, USA). The tri-angular PyrexNitride AFM probes (PNP-TR-20) were purchased from NanoWorld (Neuchâtel, Switzerland). The AFM Calibration block (TGZ1) characterized by a step height of $20 \pm 1.5 \mathrm{~nm}$ was purchased from Ted Pella Inc. (Redding, CA, USA). Fluorescein isothiocyanate dextran MW markers, 4 kDa (FD), as well reagent grade salts were purchased from Sigma Aldrich (Saint Louis, MO, USA).

\subsection{Methods}

\subsubsection{Baseline Diffusion Measurements}

Baseline diffusion measurements were carried out to ensure porosity of the synthetic membranes prior to AFM measurements. Calibration stock solutions of $5 \mathrm{mg} / \mathrm{mL}$ for each FITC-Dextran MW standard (4 kDa or Stokes' radii of $1.4 \mathrm{~nm}$ ) dissolved in $0.9 \%(w / v)$ $\mathrm{NaCl}$ were prepared. In dilute solutions, there is a linear relationship between absorbance and the concentration of the marker under observation, thus diffusion measurements were carried out at this nominal concentration. Dialysis tubing of various MW cutoffs were filled with $2 \mathrm{~mL}$ of MW marker solutions and subsequently incubated and stirred at $120 \mathrm{rpm}$ in a $10 \mathrm{~mL}$ beaker filled with $0.9 \%(w / v) \mathrm{NaCl}$. The supernatant absorbance was measured prior to $\left(\mathrm{A}_{0}\right)$ and post-mixing at $350 \mathrm{~nm}$ using a Genesis $10 \mathrm{~S}$ UV-Vis spectrophotometer (Thermo Fisher Scientific, Waltham, MA, USA). The absorbance value at $8 \mathrm{~h}$ (A) was taken to be the equilibrium value.

The molecular weight cutoff (MWCO) of the membrane expressed in terms of Stokes' radius $\left(r_{p}\right)$ was given by Equation (1) [45]. This equation assumes that the solute of molecular weight (MW) is a sphere with a density $\left(\rho=1 \mathrm{~g} \cdot \mathrm{cm}^{-3}\right)$ equal to that of the solute in solid phase.

$$
r_{p}=\left(\frac{3 M_{w}}{4 \rho \pi N_{A}}\right)^{1 / 3}
$$

\subsubsection{Atomic Force Microscopy}

Imaging was performed via a 3100 Dimension atomic force microscopy machine (DAFM-XYZ, Bruker Instruments, Billerica, MA, USA). The atomic force microscopy (AFM) scan was conducted in tapping and fluid modes using a Pyrex-Nitride probe (PNP-TR-20, NanoWorld, Neuchâtel, Switzerland) with a triangular cantilever (resonant frequency $17 \mathrm{kHz}$, force constant $0.08 \mathrm{~N} / \mathrm{m}$, thickness $500 \mathrm{~nm}$, length $200 \mu \mathrm{m}$, tip radius 7-10 nm). A total of 6 tips were used, corresponding to the respective MW marker/imaging mode outlined in Table 6. Nanoscope v6.13 (Bruker Instruments, Billerica, MA, USA) and Gwyddion v2.3 (Czech Metrology Institute, Brno, Czechoslovakia) were used as image analysis software, respectively.

Scan speed was established by setting a ratio of 512 pixels/line for a range of frequency of $0.1-0.2 \mathrm{~Hz}$. The scan area ranged from 1 to $8.0 \mu \mathrm{m}^{2}$. The pore sizes were feature-extracted 
at a constant scan area of $1 \mu \mathrm{m}^{2}$. Images were obtained at 5 different locations for the tapping and 4 different locations for the fluid modes respectively, across the sample.

The dialysis tubing, stored in sodium azide to avoid biofouling, was cut in order to produce a $1 \mathrm{~cm}^{2}$ single ply sheath. For the tapping mode, samples were washed and allowed to equilibrate in filtered saline $(0.9 \% w / v \mathrm{NaCl})$ for $30 \mathrm{~min}$. For the fluid mode, the sample remained submerged in the saline throughout the scan.

\subsubsection{Detection of Instrument and Environmental Drifts}

Feature-extracted pore size measurements were fitted to Fourier transforms by using the nonlinear least-squares formulation of Matlab2020a with and without robust weighing options. The resultant output in terms of the adjusted coefficient of determination $\left(R_{a d j}^{2}\right)$ was used to detect periodicity in data caused by hypothetical instrument drifts during imaging for the sections under observation.

Changes in environmental conditions by sample scan location were assessed by the non-parametric Kruskal-Wallis test, equivalent to a one-way analysis of variance (ANOVA) for non-normally distributed data using the Matlab2020a kruskalwallis function at the $95 \%$ confidence interval (CI) [46].

\subsubsection{Classifier Model Development}

Normality Assessment and Hypothesis Testing for Inter MWCO Discrimination

Normality was quantitatively assessed using the Jarque-Bera test [47] combining the central distribution moments given by Equation (2):

$$
J B=n\left(\frac{\left(\sqrt{b_{1}}\right)^{2}}{6}+\frac{\left(b_{2}-3\right)^{2}}{24}\right)
$$

where $b_{1}$ and $b_{2}$ are the sample skewness and sample excess kurtosis, $n$ is the sample size and $J B$ is the Jarque-Bera metric, respectively. The $J B$ test statistic is approximately Chi-squared $\left(\chi^{2}\right)$-distributed, under the assumption that the null hypothesis is true. It is equal to zero when the distribution has zero skewness and kurtosis is 3.

Hypothesis testing using parametric and non-parametric methods was carried out to assess whether the RC membranes may be distinguished based on mean/median values at the $95 \%$ CI. If the data were normally distributed, hypothesis testing was used, using a two-sample t-test with the Matlab2020a ttest2 function. Homoscedasticity was conducted using the Levene test (Matlab2020a vartestn) function prior to conducting the two-way comparisons at the $95 \% \mathrm{CI}$ to compare the mean pore size distributions [46]. If the data were not normally distributed, the Kruskal-Wallis test was used at the 95\% CI.

\section{Data Transformation}

Pending negative normality, the data were fitted to the Gamma distribution using the Matlab2020a gamfit function, in order to address the skewness of the raw data. Random variable $X$ has a Gamma distribution if its probability distribution function is given by Equation (3), where $a$ and $b(a>0$ and $b>0)$ are respectively the shape parameter and scale parameter [46]:

$$
f(x)=\frac{1}{b^{a} \Gamma(a)} x^{a-1} e^{-\frac{x}{b}}, \quad x \geq 0
$$

Logistic Regression and Receiver Operating Characteristic Curves

Following distribution parameter determination for the Gamma distribution, a Monte Carlo simulation was used to generate 10 datasets for each membrane type/imaging mode pair. Logistic regression (Equation (4)) was conducted on the datasets using a constant $80 \%$ of the data for training and $20 \%$ for testing, for the randomly generated datasets. The 
binomial link function is provided between the probability, $p$, and the covariate, $x$, for a binomially distributed variable:

$$
\operatorname{logit}\left(p_{i}\right)=\log \frac{p_{i}}{1-p_{i}}=\beta_{0}+\beta_{1} x_{i}, i=1, \ldots, k, \sum_{i=1}^{k} n_{i}=n
$$

ROC curves were generated using the logistic link function. Discrimination strength in terms of the area under the curve (AUC) was evaluated qualitatively using the categorical scale presented in Table 9.

Table 9. Categorical scale for AUC.

\begin{tabular}{cc}
\hline Strength & AUC \\
\hline high & $0.8-1$ \\
\hline medium & $0.7-0.8$ \\
\hline low & $<0.7$ \\
\hline
\end{tabular}

A validation step was conducted by comparing the output of the software WEKA (Waikato Environment for Knowledge Analysis, University of Waikato, New Zealand) in terms of relative error of AUCs with an acceptable limit of $10 \%$ and below.

\section{Cross-Validation and Optimal Testing Sample Size Determination}

Following algorithm validation, the size of the bootstrapped training set was subsequently varied from $10 \%$ to $100 \%$ according to the sample hold-out method in order to assess the effect of sample size reduction on AUC. The two-fold optimization criteria for the size are a minimal rate of change for the average value and an AUC $>0.7$ after subtracting the standard deviation from the mean value.

The logistic regression parameters evaluated at the optimal Youden's index $\left(Y_{i}\right)$ and the corresponding classification accuracy values are reported. The Youden's index was calculated using Equation (5), where $\left(S e_{i}\right)$ and $\left(S p_{i}\right)$ are the sensitivity and specificity at each cutoff, respectively [46]:

$$
Y_{i}=\max \left(\frac{S e_{i}+S p_{i}-1}{\sqrt{2}}\right)
$$

Classification accuracy $(A c c)$, given by Equation (6), is defined as the rate of correct classification, where $(T P)$ and $(T N)$ are the true positives and true negatives respectively, and $(N)$ is total number of classified samples.

$$
A c c=\left(\frac{T P+T N}{N}\right)
$$

\section{Conclusions}

Using supervised learning, the specific aims of pore size classification and reduction of scanning time by predictive modeling have been met. Use of RC membrane standards enabled detection of instrument accuracy errors associated with the AFM spatial resolution, thus enhancing the classification quality. Higher measurement accuracies are achieved in tapping mode. Resonance challenges created by pure hydrodynamic forces need to be decoupled from those added from sample tip contamination in order to quantify the bias between the average pore sizes reported for each method. Results of non-parametric analyses and logistic regression models coupled to projected hydrogel swelling analysis point to a loss of instrument resolution below $5 \mathrm{~nm}$. With a hypothesized strong correlation of test size to scan time, in the future, researchers may shorten the experiment time for the fluid and tapping modes accordingly, and dedicate the saved time to replication. Future 
efforts will explore the use of FM-AFM capabilities, tip treatment and a dew point controller in order to improve the lower limit of detection, followed by the subsequent validation using the proposed classifier algorithms. Of particular interest is to establish a framework for the predictive analysis of pore sizes obtained using scanning probe microscopy from biomimetic anisotropic materials [48,49]. Under enhanced capture methods for improving spatial resolution, parallel supervised learning techniques namely support vector machines will be run in parallel and cross-validated to compare the extent of overfitting as well as examining the nature of the outliers contributing to non-Gaussian data [28].

Supplementary Materials: The following are available online at https://www.mdpi.com/article/ 10.3390/ma14216724/s1, Figure S1: AFM scan corresponding to the calibration block. The step height for the calibration standard was measured to be $21.4 \pm 1.5 \mathrm{~nm}$ and a period (pitch) of $3 \pm 0.01 \mu \mathrm{m}$. Figure S2A: Raw data for AFM pore size captured (T1-T3 tapping mode; F1-F3 fluid mode) chronologically across the examined sections ( 5 sections for tapping mode, 4 sections for fluid mode), showing random spikes across all 3 examined RC membranes. The data were subjected to the Fourier analysis for the detection of periodic instrument and environmental drifts ruled out due to poor adjusted coefficients of determination $\left(R_{a d j}^{2}<<1\right)$. Results are summarized in Table 3 . Figure S2B: Pore size frequency distribution (T4-T6 tapping mode; F4-F6 fluid mode) indicative of lack of normality. Figure S2C: Raw datasets fitted to Gamma distributions (Figure S2, T7 tapping mode; Figure S2, F7 fluid mode). Figure S3: Receiver operating curves applied to the raw datasets. Top and bottom rows correspond to tapping and fluid modes, respectively: (a,d) $100 \mathrm{kDa}$ vs. $1000 \mathrm{kDa}$ in tapping and fluid modes, (b,e) $50 \mathrm{kDa}$ vs. $1000 \mathrm{kDa}$ in tapping and fluid modes, (c,f) $50 \mathrm{kDa}$ vs. $100 \mathrm{kDa}$ in tapping and fluid modes. Corresponding AUCs are presented in Table 8.

Author Contributions: A.H. conducted the supervised learning analysis and contributed to manuscript preparation; H.C. designed and conducted all AFM measurements and contributed to data analysis; R.B.J. co-supervised H.C. and critically reviewed and edited the manuscript for AFM robustness to measurement uncertainties content; U.K. co-supervised H.C. and critically reviewed and edited the manuscript from a sensor reliability perspective; M.M.-M. conceived the study, conducted the formal analysis, co-supervised A.H. and H.C., co-wrote the manuscript and critically reviewed it for biomaterials and statistical learning content. All authors have read and agreed to the published version of the manuscript.

Funding: This research was funded by the Bannan School of Engineering Clare Boothe Luce Funds and Department of Bioengineering at Santa Clara University.

Acknowledgments: The authors would like to acknowledge Shaun Park Snyder for her expert advice and mentorship throughout the work.

Conflicts of Interest: The authors declare no conflict of interest.

\section{References}

1. Vitas, S.; Segmehl, J.; Burgert, I.; Cabane, E. Porosity and pore size distribution of native and delignified beech wood determined by mercury intrusion porosimetry. Materials 2019, 12, 416. [CrossRef] [PubMed]

2. Miyashiro, D.; Hamano, R.; Umemura, K. A review of applications using mixed materials of cellulose, nanocellulose and carbon nanotubes. Nanomaterials 2020, 10, 186. [CrossRef]

3. Martoïa, F.; Dumont, P.J.; Orgéas, L.; Belgacem, M.N.; Putaux, J.-L. On the origins of the elasticity of cellulose nanofiber nanocomposites and nanopapers: A micromechanical approach. RSC Adv. 2016, 6, 47258-47271. [CrossRef]

4. Meng, Q.; Shi, X. A microstructure-based constitutive model of anisotropic cellulose nanopaper with aligned nanofibers. Extrem. Mech. Lett. 2021, 43, 101158. [CrossRef]

5. Curvello, R.; Raghuwanshi, V.S.; Garnier, G. Engineering nanocellulose hydrogels for biomedical applications. Adv. Colloid Interface Sci. 2019, 267, 47-61. [CrossRef] [PubMed]

6. Yang, Y.; Lu, Y.T.; Zeng, K.; Heinze, T.; Groth, T.; Zhang, K. Recent progress on cellulose-based ionic compounds for biomaterials. Adv. Mater. 2020, 33, 2000717. [CrossRef] [PubMed]

7. Kim, S.; Kang, J.Y.; Balance, W.C.; Sutton, B.P.; Shin, D.H.; Jang, K.H.; Shin, M.; Kong, H.; Kim, J.W. Fabrication of cell penetrating peptide-conjugated bacterial cellulose nanofibrils with remarkable skin adhesion and water retention performance. Int. J. Pharm. 2021, 600, 120476. [CrossRef]

8. Mineshima, M. The past, present and future of the dialyzer. Contrib. Nephrol. 2015, 185, 8-14. [PubMed]

9. Junter, G.-A.; Lebrun, L. Cellulose-based virus-retentive filters: A review. Rev. Environ. Sci. Biotechnol. 2017, 16, 455-489. [CrossRef] 
10. Ma, Z.; Ramakrishna, S. Electrospun regenerated cellulose nanofiber affinity membrane functionalized with protein a/g for igg purification. J. Membr. Sci. 2008, 319, 23-28. [CrossRef]

11. Östlund, Å.; Idström, A.; Olsson, C.; Larsson, P.T.; Nordstierna, L. Modification of crystallinity and pore size distribution in coagulated cellulose films. Cellulose 2013, 20, 1657-1667. [CrossRef]

12. Zhou, J.; Zhang, L.; Cai, J.; Shu, H. Cellulose microporous membranes prepared from Naoh/Urea aqueous solution. J. Membr. Sci. 2002, 210, 77-90. [CrossRef]

13. Simpliciano, C.; Clark, L.; Asi, B.; Chu, N.; Mercado, M.; Diaz, S.; Goedert, M.; Mobed-Miremadi, M. Cross-linked alginate film pore size determination using atomic force microscopy and validation using diffusivity determinations. J. Surf. Eng. Mater. Adv. Technol. 2013, 3, 1-12. [CrossRef]

14. Feger, G.; Angelov, B.; Angelova, A. Prediction of amphiphilic cell-penetrating peptide building blocks from protein-derived amino acid sequences for engineering of drug delivery nanoassemblies. J. Phys. Chem. B 2020, 124, 4069-4078. [CrossRef]

15. Wan, S.; Liang, X.; Jiang, H.; Sun, J.; Djilali, N.; Zhao, T. A coupled machine learning and genetic algorithm approach to the design of porous electrodes for redox flow batteries. Appl. Energy 2021, 298, 117177. [CrossRef]

16. Yu, H.; Chen, G.; Gu, H. A machine learning methodology for multivariate pore-pressure prediction. Comput. Geosci. 2020, 143, 104548. [CrossRef]

17. Krishnapriyan, A.S.; Montoya, J.; Haranczyk, M.; Hummelshøj, J.; Morozov, D. Machine learning with persistent homology and chemical word embeddings improves prediction accuracy and interpretability in metal-organic frameworks. Sci. Rep. 2021, 11, 1-11. [CrossRef]

18. Özkan, M.; Borghei, M.; Karakoç, A.; Rojas, O.J.; Paltakari, J. Films based on crosslinked tempo-oxidized cellulose and predictive analysis via machine learning. Sci. Rep. 2018, 8, 1-9. [CrossRef]

19. Müller, P.; Abuhattum, S.; Möllmert, S.; Ulbricht, E.; Taubenberger, A.V.; Guck, J. Nanite: Using machine learning to assess the quality of atomic force microscopy-enabled nano-indentation data. BMC Bioinform. 2019, 20, 1-9. [CrossRef] [PubMed]

20. Liu, Y.; Sun, Q.; Lu, W.; Wang, H.; Sun, Y.; Wang, Z.; Lu, X.; Zeng, K. General resolution enhancement method in atomic force microscopy using deep learning. Adv. Theory Simul. 2018, 2, 1800137. [CrossRef]

21. Oinonen, N. Interpreting Atomic Force Microscope Images with Machine Learning. Available online: https://aaltodoc.aalto.fi/ handle/123456789/39948 (accessed on 14 August 2021).

22. Mencattini, A.; Casti, P.; Fazio, G.; Ghibelli, L.; Luce, M.; Cricenti, A.; Martinelli, E.; Natale, C.D. Uncertainty evaluation of a Vbm system for Afm study of cell-cerium oxide nanoparticles interactions. IEEE Trans. Instrum. Meas. 2018, 67, 1564-1572. [CrossRef]

23. Minelli, E.; Ciasca, G.; Sassun, T.E.; Antonelli, M.; Palmieri, V.; Papi, M.; Maulucci, G.; Santoro, A.; Giangaspero, F.; Delfini, R.; et al. A fully-automated neural network analysis of AFM force-distance curves for cancer tissue diagnosis. Appl. Phys. Lett. 2017, 111, 143701. [CrossRef]

24. Sokolov, I.; Dokukin, M.E.; Kalaparthi, V.; Miljkovic, M.; Wang, A.; Seigne, J.D.; Grivas, P.; Demidenko, E. noninvasive diagnostic imaging using machine-learning analysis of nanoresolution images of cell surfaces: Detection of bladder cancer. Proc. Natl. Acad. Sci. USA 2018, 115, 12920-12925. [CrossRef] [PubMed]

25. Huang, B.; Li, Z.; Li, J. An artificial intelligence atomic force microscope enabled by machine learning. Nanoscale 2018, 10, 21320-21326. [CrossRef] [PubMed]

26. Borodinov, N.; Tsai, W.-Y.; Korolkov, V.V.; Balke, N.; Kalinin, S.V.; Ovchinnikova, O.S. Machine learning-based multidomain processing for texture-based image segmentation and analysis. Appl. Phys. Lett. 2020, 116, 044103. [CrossRef]

27. Sundstrom, A.; Cirrone, S.; Paxia, S.; Hsueh, C.; Kjolby, R.; Gimzewski, J.K.; Reed, J.; Mishra, B. Image analysis and length estimation of biomolecules using AFM. IEEE Trans. Inf. Technol. Biomed. 2012, 16, 1200-1207. [CrossRef]

28. James, G.; Witten, D.; Hastie, T.J.; Tibshirani, R. An Introduction to Statistical Learning; Springer: New York, NY, USA, 2017.

29. Berthold, T.; Benstetter, G.; Frammelsberger, W.; Rodríguez, R.; Nafría, M. Numerical study of hydrodynamic forces for AFM operations in liquid. Scanning 2017, 2017, 1-12. [CrossRef] [PubMed]

30. Colson, J.; Andorfer, L.; Nypelö, T.E.; Lütkemeier, B.; Stöckel, F.; Konnerth, J. Comparison of silicon and OH-Modified AFM tips for adhesion force analysis on functionalised surfaces and natural polymers. Colloids Surf. A Physicochem. Eng. Asp. 2017, 529, 363-372. [CrossRef]

31. Gusenbauer, C.; Nypelö, T.; Jakob, D.S.; Xu, X.G.; Vezenov, D.V.; Asaadi, S.; Sixta, H.; Konnerth, J. Differences in surface chemistry of regenerated lignocellulose fibers determined by chemically sensitive scanning probe microscopy. Int. J. Biol. Macromol. 2020, 165, 2520-2527. [CrossRef]

32. Zhu, Q.; Zhou, R.; Liu, J.; Sun, J.; Wang, Q. Recent Progress on the Characterization of Cellulose Nanomaterials by Nanoscale Infrared Spectroscopy. Available online: https:/ /www.ncbi.nlm.nih.gov/pmc/articles/PMC8190638/ (accessed on 19 October 2021).

33. Wagner, R.; Moon, R.J.; Raman, A. Mechanical Properties of Cellulose Nanomaterials Studied by Contact Resonance Atomic Force Microscopy. Available online: https:/ /link.springer.com/article/10.1007/s10570-016-0883-4 (accessed on 19 October 2021).

34. Lo, Y.-S.; Huefner, N.D.; Chan, W.S.; Dryden, P.; Hagenhoff, B.; Beebe, T.P. Organic and inorganic contamination on commercial AFM cantilevers. Langmuir 1999, 15, 6522-6526. [CrossRef]

35. Kabir, S.M.; Sikdar, P.P.; Haque, B.; Bhuiyan, M.A.; Ali, A.; Islam, M.N. Cellulose-based hydrogel materials: Chemistry, properties and their prospective applications. Prog. Biomater. 2018, 7, 153-174. [CrossRef]

36. Okugawa, A.; Sakaino, M.; Yuguchi, Y.; Yamane, C. Relaxation phenomenon and swelling behavior of regenerated cellulose fibers affected by water. Carbohydr. Polym. 2020, 231, 115663. [CrossRef] 
37. Ye, D.; Zhong, Z.; Xu, H.; Chang, C.; Yang, Z.; Wang, Y.; Ye, Q.; Zhang, L. Construction of cellulose/nanosilver sponge materials and their antibacterial activities for infected wounds healing. Cellulose 2015, 23, 749-763. [CrossRef]

38. Fluid Imaging AFM of Cellulose Bio-Membranes. Available online: https://www.eag.com/resources/appnotes/fluid-imagingafm-of-cellulose-bio-membranes / (accessed on 19 October 2021).

39. Moiseev, Y.N.; Mostepanenko, V.M.; Panov, V.I.; Sokolov, I.Y. Force dependences for the definition of the atomic force microscopy spatial resolution. Phys. Lett. A 1988, 132, 354-358. [CrossRef]

40. Gan, Y. Atomic and subnanometer resolution in ambient conditions by atomic force microscopy. Surf. Sci. Rep. 2009, 64, 99-121. [CrossRef]

41. Kuwahara, S.; Akita, S.; Shirakihara, M.; Sugai, T.; Nakayama, Y.; Shinohara, H. Fabrication and characterization of high-resolution AFM tips with high-quality double-wall carbon nanotubes. Chem. Phys. Lett. 2006, 429, 581-585. [CrossRef]

42. Fukuma, T.; Kobayashi, K.; Matsushige, K.; Yamada, H. True molecular resolution in liquid by frequency-modulation atomic force microscopy. Appl. Phys. Lett. 2005, 86, 193108. [CrossRef]

43. Baranwal, M.; Gorugantu, R.S.; Salapaka, S.M. Robust atomic force microscopy using multiple sensors. Rev. Sci. Instrum. 2016, 87, 083704. [CrossRef]

44. Akrami, S.M.; Nakayachi, H.; Watanabe-Nakayama, T.; Asakawa, H.; Fukuma, T. Significant improvements in stability and reproducibility of atomic-scale atomic force microscopy in liquid. Nanotechnology 2014, 25, 455701. [CrossRef]

45. Fournier, R.L. Basic Transport Phenomena in Biomedical Engineering; CRC Press: Boca Raton, FL, USA, 2012.

46. Vidakovic, B. Engineering Biostatistics: An Introduction Using Matlab and WinBUGS; Wiley: Hoboken, NJ, USA, 2017.

47. Yap, B.W.; Sim, C.H. Comparisons of various types of normality tests. J. Stat. Comput. Simul. 2011, 81, 2141-2155. [CrossRef]

48. Despang, F.; Dittrich, R.; Gelinsky, M. Novel biomaterials with parallel aligned pore channels by directed ionotropic gelation of alginate: Mimicking the anisotropic structure of bone tissue. Adv. Biomim. 2011, 349-372.

49. Oprea, M.; Voicu, S.I. Recent advances in applications of cellulose derivatives-based composite membranes with hydroxyapatite. Materials 2020, 13, 2481. [CrossRef] [PubMed] 